\title{
ISOLATED SINGULARITIES OF MONGE-AMPÈRE EQUATIONS
}

\author{
FRIEDMAR SCHULZ AND LIHE WANG
}

(Communicated by Palle E. T. Jorgensen)

\begin{abstract}
In this paper, we give conditions which ensure that isolated singularities of solutions of the elliptic Monge-Ampère equation $\operatorname{det} D^{2} u=1$ are removable.
\end{abstract}

\section{INTRODUCTION}

We use the notation $D^{2} u$ for the matrix of second-order derivatives of the function $u=u(x)$. Furthermore, for a point $x$ in $\mathbf{R}^{n},|x|$ is the norm, $e_{1}, \ldots, e_{n}$ are the unit vectors in the direction of the coordinate axis, and $B_{r}=\{x:|x|<r\}$ is the ball of radius $r$ centered at the origin 0 .

For the statement of the main theorem of this paper, let $u$ be defined in the punctured ball $B_{2} \backslash\{0\}$.

Theorem 1. Suppose that $u$ is a smooth convex solution of the elliptic MongeAmpère equation

$$
\operatorname{det} D^{2} u=1
$$

in $B_{2} \backslash\{0\}$. Then $u$ has a locally Lipschitz continuous extension to $B_{2}$ which is smooth if and only if it is $C^{1}$ along a line through the origin 0 .

This theorem was proven by $\mathrm{K}$. Jörgens [J] in 1955 in the two-dimensional case. Recently, R. Beyerstedt [B] extended Jörgens' theorem to more general Monge-Ampère equations, also in the case $n=2$.

We remark that the convexity condition is redundant in the two-dimensional case. In a future work, we intend to show how our multidimensional methods apply to much more general fully nonlinear equations.

\section{Convexity}

Lemma 2. Every convex function $u$ in $B_{1} \backslash\{0\}$ has a convex extension to $B_{1}$. It is therefore locally Lipschitz continuous, and $\frac{\partial u}{\partial e}(0)$ exists for any unit vector e. Furthermore,

$$
\frac{\partial u}{\partial(-e)}(0) \geq-\frac{\partial u}{\partial e}(0),
$$

Received by the editors May 4, 1994 and, in revised form, May 16, 1994.

1991 Mathematics Subject Classification. Primary 35Q99, 35 J60.

Key words and phrases. PDE, nonlinear, elliptic, Monge-Ampère, singularity.

The second author was supported by the National Science Foundation. 
$\frac{\partial u}{\partial e}(t e)$ is monotone non-decreasing in $t$ and continuous from the right, i.e.,

$$
\lim _{t \rightarrow 0^{+}} \frac{\partial u}{\partial e}(t e)=\frac{\partial u}{\partial e}(0) \text {. }
$$

The proof is elementary. The listed properties hold for any $x \in B_{1}$; and the arguments are simpler, if $u$ is $C^{1}$ in $B_{1} \backslash\{0\}$.

Under this assumption, $u$ is $C^{1}$ along the line in the direction $e$ through the origin, iff

$$
\frac{\partial u}{\partial(-e)}(0)=-\frac{\partial u}{\partial e}(0) \text {. }
$$

By thickening the line, one can then argue that this is the case iff $\frac{\partial u}{\partial e}$ is $C^{0}$ in $B_{1}$, because $\frac{\partial u}{\partial e}$ is monotone on all lines in the direction $e$.

The following theorem is of some interest in its own right. For simplicity, we only consider the two-dimensional case, because this $C^{1}$-regularity result is not needed in the proof of Theorem 1.

Proposition 3. Let $u$ be a convex function in $B_{1} \subset \mathbf{R}^{2}$. Suppose that $\frac{\partial u}{\partial\left(-e_{1}\right)}(0)=$ $-\frac{\partial u}{\partial e_{1}}(0)$ and that $u$ is $C^{1}$ in $B_{1} \backslash\{0\}$. Then $\frac{\partial u}{\partial e}$ is $C^{0}$ in the sector $S_{t}=$ $\left\{(x, y) \in B_{1}: y \geq t|x|\right\}$ for any unit vector $e=(a, b), b \geq 0$, and any $t>0$.

Proof. First, by subtracting a linear function, we may assume that $u(0,0)=0$, $\frac{\partial u}{\partial x}(0,0)=0, \frac{\partial u}{\partial e_{2}}(0,0)=0$.

By convexity, for $(x, y) \in B_{1 / 2}, y>0$,

$$
u(x, y) \leq \begin{cases}\frac{x u(x+y, 0)+y u(0, x+y)}{x+y} & (x>0), \\ \frac{x u(x-y, 0)-y u(0, y-x)}{x-y} & (x<0) .\end{cases}
$$

Note that the function on the LHS extends $u(x, 0)$ and $u(0, y)$ linearly in the directions $e_{1} \mp e_{2}$. Hence

$$
\frac{\partial u}{\partial e}(0,0) \leq a \frac{\partial u}{\partial e_{1}}(0,0)+b \frac{\partial u}{\partial e_{2}}(0,0)=0
$$

for all $e=(a, b), b \geq 0$.

On the other hand,

$$
u(0, y) \leq \frac{u(x, y)+u(-x, y)}{2}
$$

for all $(x, y) \in B_{1}$, which implies that

$$
0 \leq 2 b \frac{\partial u}{\partial e_{2}}(0,0) \leq \frac{\partial u}{\partial e}(0,0)+\frac{\partial u}{\partial e^{-}}(0,0),
$$

where $e^{-}=(-a, b)$. Therefore

$$
\frac{\partial u}{\partial e}(0,0)=0
$$

for all $e=(a, b), b \geq 0$. It follows that

$$
\frac{\partial u}{\partial(-e)}(0,0) \geq 0,
$$

and in turn that $u \geq 0$ in $B_{1}$. 
For $t>0$, consider the direction $e_{t}=(a, b), b=t a>0$. Then, by the monotonicity of $\frac{\partial u}{\partial e_{t}}$ on all lines in the direction $e_{t}$, for any given $\varepsilon<0$, there is a $\delta>0$ such that

$$
\left|\frac{\partial u}{\partial e_{t}}\right| \leq \varepsilon
$$

in the parallelogram $\left\{(x, y) \in B_{1}: 0 \leq x \leq a, t x \leq y \leq t x+\delta\right\}$.

A similar argument can be made for $e_{t}^{-}$, which implies that $\frac{\partial u}{\partial e}$ is $C^{0}$ in $S_{t}$, for any $e=(a, b), b \geq 0$, as required.

\section{THE COMPARISON PRINCIPLE}

We show that $u$ satisfies the comparison principle. In this section, we use the notation $B_{r}(x)=x+B_{r}$.

Lemma 4. Let $v_{1}$ and $v_{2}$ be $C^{2}$ in $B_{1 / 2}\left(\frac{1}{2} e_{1}\right)$ and $C^{0}$ in $\overline{B_{1 / 2}\left(\frac{1}{2} e_{1}\right)}$, which solve the equation

$$
\operatorname{det} D^{2} u=1 \text { in } B_{1 / 2}\left(\frac{1}{2} e_{1}\right) \text {. }
$$

Assume further that $v_{1}(0)=v_{2}(0), v_{2} \geq v_{1}$ on $\partial B_{1 / 2}\left(\frac{1}{2} e_{1}\right)$, and $v_{1} \not \equiv v_{2}$. Then

(a) $v_{2} \geq v_{1}$ in $B_{1 / 2}\left(\frac{1}{2} e_{1}\right)$.

(b) If we assume further that $v_{1}$ and $v_{2}$ are $C^{2}$ in $\overline{B_{1 / 2}\left(\frac{1}{2} e_{1}\right)}$, then

$$
\frac{\partial v_{2}}{\partial x_{1}}(0)>\frac{\partial v_{1}}{\partial x_{1}}(0)
$$

Proof. (a) follows from the weak maximum principle [GT], Theorem 17.1, page 443, which in turn follows from the classical weak maximum principle [GT], Theorem 3.1.

To prove (b), let $w=v_{2}-v_{1}$. Then

$$
\sum_{i, j=1}^{n} A_{i j}(x) \frac{\partial^{2} w}{\partial x_{i} \partial x_{j}}=\operatorname{det} D^{2} v_{2}-\operatorname{det} D^{2} v_{1}=0
$$

where $A_{i j}(x)=\partial \operatorname{det}\left(\theta D^{2} v_{2}+(1-\theta) D^{2} v_{1}\right) / \partial u_{i j}$ for some $\theta=\theta(x), 0<\theta<$ 1 , which depends on $D^{2} v_{1}$ and $D^{2} v_{2}$. Since $\left[A_{i j}\right]$ is uniformly elliptic, the classical strong maximum principle [GT], Theorem 3.5, together with the Hopf lemma [GT], Lemma 3.4, yield statement (b).

Lemma 5. Let $v_{1}$ and $v_{2}$ be as in Lemma 4. Suppose further that $v_{2}$ is $C^{3}$ in $\overline{B_{1 / 2}\left(\frac{1}{2} e_{1}\right)}$ and that $v_{2}\left(e_{1}\right)>v_{1}\left(e_{1}\right)$. Then $\frac{\partial v_{2}}{\partial x_{1}}(0)>\frac{\partial v_{1}}{\partial x_{1}}(0)$.

Proof. Let $\varphi_{2}$ be a smooth function such that $\varphi_{2}=v_{2}$ on $\partial B_{1 / 2}\left(\frac{1}{2} e_{1}\right) \cap B_{1 / 2}(0)$, $v_{1}<\varphi_{2}<v_{2}$ near $e_{1}$, and $v_{1} \leq \varphi_{2} \leq v_{2}$ on $\partial B_{1 / 2}\left(\frac{1}{2} e_{1}\right)$. Let $u_{2}$ be the solution of

$$
\begin{cases}\operatorname{det} D^{2} u_{2}=1 & \text { in } B_{1 / 2}\left(\frac{1}{2} e_{1}\right), \\ u_{2}=\varphi_{2} & \text { on } \partial B_{1 / 2}\left(\frac{1}{2} e_{1}\right),\end{cases}
$$

whose existence follows from [GT], Theorem 17.22, page 473, in combination with Problem 17.11 (ii), page 490. By Lemma $5, v_{2} \geq u_{2} \geq v_{1}$ in $B_{1 / 2}\left(\frac{1}{2} e_{1}\right)$.

Moreover,

$$
\frac{\partial v_{2}}{\partial x_{1}}(0)>\frac{\partial u_{2}}{\partial x_{1}}(0)
$$


Hence

$$
\frac{\partial v_{2}}{\partial x_{1}}(0)>\frac{\partial u_{2}}{\partial x_{1}}(0) \geq \frac{\partial v_{1}}{\partial x_{1}}(0)
$$

Proof of the main theorem. Let $v$ be the solution of

$$
\begin{cases}\operatorname{det} D^{2} v=1 & \text { in } B_{1} \\ v=u & \text { on } \partial B_{1}(0)\end{cases}
$$

The claim is that $u=v$. We only show $v \geq u$ by contradiction, since the other part is similar.

Let $\varepsilon$ be the minimum such that

$$
v \geq u-\varepsilon \text { in } \overline{B_{1}(0)} \text {. }
$$

By the assumption, $\varepsilon>0$ and $v\left(x_{0}\right)=u\left(x_{0}\right)-\varepsilon$ for some $x_{0} \in \overline{B_{1}(0)}$.

We may assume that $x_{0}=0$, because otherwise the classical strong maximum principle implies that $v \equiv u-\varepsilon$, which is a contradiction. If $x_{0}=0$, then $\frac{\partial v}{\partial x_{1}}(0)=\frac{\partial u}{\partial x_{1}}(0)$, since we may assume that $u$ is $C^{1}$ along the $x_{1}$-axis.

However, Lemma 5 now implies that

$$
\frac{\partial v}{\partial x_{1}}(0)>\frac{\partial u}{\partial x_{1}}(0)
$$

which is a contradiction.

\section{REFERENCES}

[B] R. Beyerstedt, Removable singularities of solutions to elliptic Monge-Ampère equations, Math. Z. 208 (1991), 363-373.

[J] K. Jörgens, Harmonische Abbildungen und die Differentialgleichung $r t-s^{2}=1$, Math. Ann. 129 (1955), 330-344.

[GT] D. Gilbarg and N. Trudinger, Elliptic partial differential equations of second order, 2nd ed., Springer-Verlag, Berlin, Heidelberg, and New York, 1983.

Mathematik I, Universität Ulm, D-89069 Ulm, Germany

E-mail address: friedmar. schulzOmathematik.uni-ulm.de

Department of Mathematics, University of Iowa, Iowa City, Iowa 52242

E-mail address: 1wang@math. uiowa.edu 